\title{
Detection of Acetaldehyde in the Esophageal Tissue among Healthy Male Subjects after Ethanol Drinking and Subsequent L-Cysteine Intake
}

\author{
Hideki Okata, ${ }^{1}$ Waku Hatta, ${ }^{1}$ Katsunori Iijima, ${ }^{2}$ Kiyotaka Asanuma, ${ }^{1}$ \\ Atsuki Tsuruya, ${ }^{3}$ Naoki Asano, ${ }^{1}$ Tomoyuki Koike, ${ }^{1}$ Shin Hamada, ${ }^{1}$ \\ Toru Nakayama, ${ }^{3}$ Atsushi Masamune ${ }^{1, *}$ and Tooru Shimosegawa ${ }^{1, *}$ \\ ${ }^{1}$ Division of Gastroenterology, Tohoku University Graduate School of Medicine, Sendai, Miyagi, Japan \\ ${ }^{2}$ Department of Gastroenterology and Neurology, Akita University Graduate School of Medicine, Akita, Akita, \\ Japan \\ ${ }^{3}$ Department of Biomolecular Engineering, Graduate School of Engineering, Tohoku University, Sendai, Miyagi, \\ Japan
}

\begin{abstract}
Ethanol is oxidized by alcohol dehydrogenase to acetaldehyde, a recognized carcinogen for the esophagus. However, no previous study has measured the acetaldehyde levels in the esophageal tissue. L-cysteine has been shown to reduce the acetaldehyde levels in the saliva; however, it is unknown whether L-cysteine intake affects the acetaldehyde concentration in the esophageal tissue. The aim of this study was to measure the acetaldehyde concentration in the esophageal tissue after ethanol drinking and evaluate the effect of L-cysteine intake on the acetaldehyde levels in the esophagus. We enrolled 10 male subjects with active acetaldehyde dehydrogenase- $2 * 1 /{ }^{*} 1\left(A L D H 2 * 1 /{ }^{*} 1\right)$ genotype and 10 male subjects with the inactive acetaldehyde dehydrogenase- $2{ }^{*} 1{ }^{*} 2\left(A L D H 2 * 1 /{ }^{*} 2\right)$ genotype, the mean ages of whom were 25.6 and 27.9 years, respectively. In this prospective, single-blind, placebo-controlled study using L-cysteine and placebo lozenges (first and second examination), saliva and blood were collected before and after ethanol drinking. Esophageal tissue was obtained by endoscopic biopsy at 60 minutes after drinking, and the acetaldehyde and ethanol concentrations were measured. The acetaldehyde concentration of the saliva was significantly lower in those taking L-cysteine than in those taking the placebo. Acetaldehyde in the esophageal tissue was detected only in those taking L-cysteine lozenges. There were no correlations between the acetaldehyde concentrations in the esophageal tissue and saliva or blood. In conclusion, we detected acetaldehyde in the human esophageal tissue after ethanol drinking. Unexpectedly, intake of L-cysteine lozenges appears to contribute to detection of acetaldehyde in the esophageal tissue.
\end{abstract}

Keywords: acetaldehyde; acetaldehyde dehydrogenase; esophageal tissue; L-cysteine; placebo. Tohoku J. Exp. Med., 2018 April, 244 (4), 317-325. C 2018 Tohoku University Medical Press

\section{Introduction}

Alcohol is a major risk factor for esophageal squamous cell carcinoma (ESCC), which represents over $90 \%$ of the esophageal cancer in Japan, and acetaldehyde, associated with alcohol intake, was added as a definite carcinogen (a group 1 carcinogen) for the esophagus by the International Agency for Research on Cancer (Secretan et al. 2009). In alcoholic metabolism, alcohol dehydrogenase (ADH) oxidizes alcohol to acetaldehyde, which is further oxidized to acetate by aldehyde dehydrogenase (ALDH) (Zakhari 2006). Thus, single nucleotide polymorphisms of $A D H$ and $A L D H$-related genes can modify the reduction of etha- nol and acetaldehyde, which would predispose heavy drinkers to cancer (Yokoyama and Omori 2005). Among these, the $A D H 1 B$ (rs1229984) and $A L D H 2$ (rs671) variations increase the incidence of ESCC, which has been demonstrated by two genome-wide association studies using Japanese cohorts (Cui et al. 2009; Tanaka et al. 2010). Therefore, variations in these genes may affect the ethanol and acetaldehyde concentrations in the esophageal tissue.

The esophageal epithelium is considered to be exposed to carcinogenic acetaldehyde via intrinsic and extrinsic pathways (Ohashi et al. 2015). In the intrinsic pathway of this theory, ethanol, which is absorbed in the upper gastrointestinal tract, is oxidized to acetaldehyde by ADH1B in

Received September 20, 2017; revised and accepted March 2, 2018. Published online April 20, 2018; doi: 10.1620/tjem.244.317.

*Senior authors.

Correspondence: Waku Hatta, Division of Gastroenterology, Tohoku University Graduate School of Medicine, 1-1 Seiryo-machi, Aobaku, Sendai, Miyagi 980-8574, Japan.

e-mail: waku-style@festa.ocn.ne.jp 
the liver, and then the excessive acetaldehyde, which is not oxidized by ALDH2 in the liver, circulates to the esophagus, saliva, and breath (Ohashi et al. 2015). In the extrinsic pathway, acetaldehyde, derived from alcohol beverages, foods, and products by the oral microflora, is directly exposed to the esophageal epithelium (Ohashi et al. 2015). Among these pathways, the locally high exposure to acetaldehyde via saliva is considered to increase the risk of cancer in the upper aerodigestive tract including the esophagus (Brooks et al. 2009; Yokoyama et al. 2010a, 2016). However, it has been difficult to measure the acetaldehyde concentration in the esophagus tissue due to the volatility, rapid metabolism, and low concentrations of acetaldehyde (Heit et al. 2016).

L-cysteine is a semi-essential amino acid that is able to reduce the carcinogenicity of acetaldehyde by forming a direct covalent bond (Salaspuro 2011), the product of which is non-toxic 2-methyl-1,3-thiazolidine-4-carboxylic acid (Sprince et al. 1975; Salaspuro et al. 2002, 2006). Preparations that slowly release L-cysteine in the oral cavity have been successfully used to reduce acetaldehyde from the saliva during alcohol consumption and smoking and from the gastric juice of patients with atrophic gastritis (Salaspuro et al. 2002, 2006; Blonigen et al. 2011). In addition, our recent study showed that intake of a slow-release L-cysteine capsule effectively reduced acetaldehyde levels in the gastric juice among active $A L D H 2 * 1 / 1$ homozygotes and inactive $A L D H 2 * 1 / * 2$ heterozygotes treated with a proton pump inhibitor (Maejima et al. 2015). Therefore, L-cysteine may also affect the acetaldehyde concentration of the esophageal tissue in each $A L D H 2$ genotype. The aim of this study was to measure acetaldehyde in human esophageal tissue and the effect of L-cysteine on acetaldehyde of the esophageal tissue in each $A L D H 2$ genotype.

\section{Methods}

\section{Subject enrollment and study design}

This prospective trial was performed between December 2015 and March 2017. In this study, we planned to enroll 20 healthy male volunteers $(10 \mathrm{ALDH} 2 * 1 / * 1$ homozygotes and $10 \mathrm{ALDH} 2 * 1 / * 2$ heterozygotes). All procedures were carried out at Tohoku University Hospital. The inclusion criteria were male 20 years or older and Helicobacter pylori (H. pylori)-negative confirmed by a ${ }^{13} \mathrm{C}$-urea breath test $\left(\mathrm{UBIT}^{\circledR}\right.$, Otsuka Pharmaceutical, Tokyo, Japan) using an infrared spectrophotometer (POCone ${ }^{\circledR}$, Otsuka Pharmaceutical). The exclusion criteria were 1) current smokers, 2) subjects after resection of gastrointestinal tract or truncal vagotomy, 3) those with ongoing therapy for any disease, 4) those taking drugs except for L-cysteine lozenges during this study, 5) those who could not drink any alcohol, 6) those with a past history of allergy for any drug, 7) those with a past history of liver, kidney or heart disease, and 8) those who were regarded as inappropriate for participating in this study by the researchers.

This was a prospective, single-blind, placebo-controlled trial using slow-release L-cysteine lozenges (Acetium lozenge ${ }^{\circledR}$, Biohit Oyj, Helsinki, Finland) and identical placebo lozenges in appearance. The study was conducted in accordance with the guidelines of the Declaration of Helsinki and was approved by the Ethics Committee for Human Research at Tohoku University School of Medicine, Sendai, Japan (2015-2-136-1, 2015-2-137-1). All subjects provided written informed consent prior to enrollment in the study. The study was registered at the University Hospital Medical Information (UMIN000019991).

\section{Study procedure}

A flowchart of the study procedure is shown in Fig. 1. At the study entry (visit 1), the eligibility including $A L D H 2$ and $A D H 1 B$ genotypes were evaluated in each subject. On two separate occasions using lozenge A (L-cysteine) and lozenge B (placebo) (visit 2 (first examination) and visit 3 (second examination)) at an interval of 1-2 months, the volunteers underwent the following procedures.

On each examination, the volunteers were instructed to refrain from consuming alcohol for 24 hours and food for 12 hours prior to this study, and they came to our institution for this study between 13 and 15 o'clock. Before drinking ethanol, $2 \mathrm{ml}$ each of saliva and blood were collected for subsequent analysis. Then, the subjects drank ethanol (Dehydrated Ethanol; Yoshida Pharmaceutical Company, Tokyo, Japan $)(0.5 \mathrm{~g} / \mathrm{kg}$ body weight $)$ diluted in orange juice to $15 \% \mathrm{w} / \mathrm{vol}$ solution in 10 minutes. This amount of alcohol corresponds to about a moderate dose of alcohol. Subsequently, a

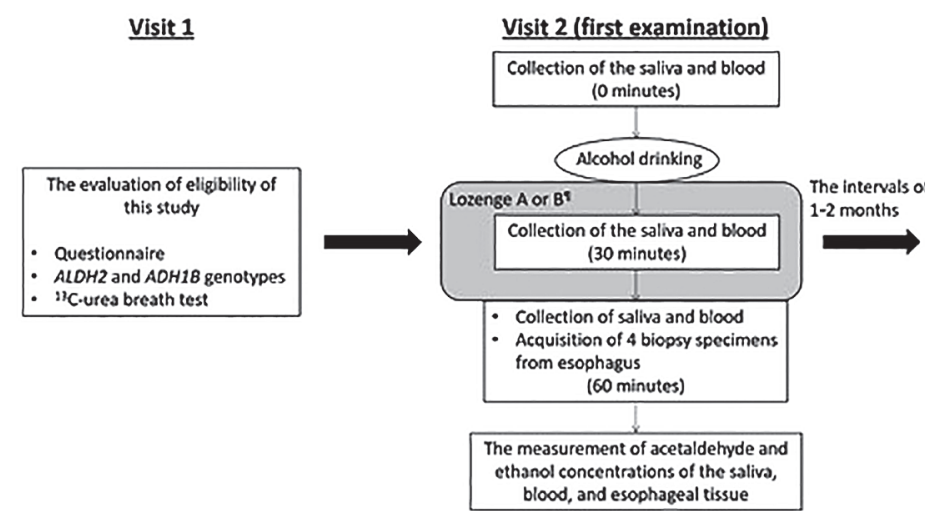

Fig. 1. The flowchart of the study procedure.

Continuous taking of lozenges for 60 minutes after drinking.

'Lozenge B or A was given to the subjects who took lozenge A or B at the first examination, respectively.

$A L D H$, acetaldehyde dehydrogenase; $A D H$, alcohol dehydrogenase.
Visit 3 (second examination)

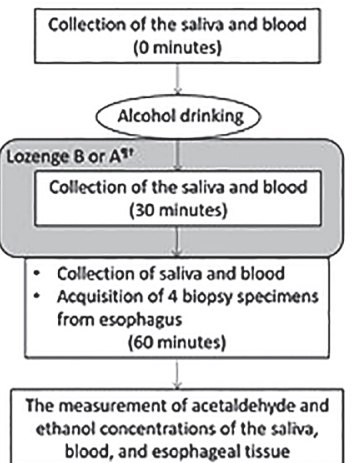

blood, and esophageal tissue 
lozenge A or B continued to be taken for 60 minutes so that these lozenges remained present in the mouth of the subjects. At 30 and 60 minutes, $2 \mathrm{ml}$ each of saliva and blood were collected. Patients were asked to stop swallowing saliva for 2 minutes before the saliva was collected. The subjects underwent esophagogastroduodenoscopy (EGD) at 60 minutes after the accumulation of lidocaine viscous oral solution 2\% (Xylocaine Viscous 2\%; AstraZeneca, Osaka, Japan) in the back of the throat for about 3 to 5 minutes as the pretreatment, and 4 biopsy specimens were taken from the esophagus $(5 \mathrm{~cm}$ above the esophagogastric junction). Before each biopsy specimen was taken, mucus on the esophageal mucosa was removed by a sufficient amount of water. The endoscopic procedure was performed by an expert endoscopist (WH). These samples were analyzed for the acetaldehyde and ethanol concentrations. The laboratory work was performed so that the investigators were blinded to the subjects' medical information, and the content of the lozenges was concealed from both subjects and researchers by the coordinator until the end of the trial.

Measurement of acetaldehyde and ethanol concentrations in the saliva, blood and esophageal tissue

A 450- $\mu 1$ sample of saliva was transferred into a headspace vial containing $50 \mu \mathrm{l}$ of $6 \mathrm{M}$ perchloric acid (PCA) to stop microbial acetaldehyde production and then frozen at $-80^{\circ} \mathrm{C}$ (Homann et al. 1997). PCA has been shown not to decompose cysteine-acetaldehyde conjugate (Blonigen et al. 2011). Blood was collected from an antecubital vein into a disposable $2.5-\mathrm{ml}$ syringe, and after immediately transferring $1 \mathrm{ml}$ of the blood into a closed vial that had been placed in ice and contained $6 \mathrm{ml}$ of a $0.6 \mathrm{M}$ PCA saline solution, it was instantly deproteinized with a test tube mixer. The precipitated proteins were centrifuged out at $2,300 \times g$ for 15 minutes at $4^{\circ} \mathrm{C}$, and a 2-ml sample of the clear supernatant was transferred to a closed headspace vial and stored at $-80^{\circ} \mathrm{C}$. Four biopsy specimens were immediately transferred into a headspace vial containing $300 \mu \mathrm{l}$ of $6 \mathrm{M}$ PCA and frozen at $-80^{\circ} \mathrm{C}$. The samples were stored at $-80^{\circ} \mathrm{C}$ until transfer on dry ice to Department of Biomolecular Engineering, Graduate School of Engineering, Tohoku University, Sendai, Japan, for further analysis. The acetaldehyde and ethanol concentrations of each sample were determined by headspace gas chromatography on an Vfwax 19091N233 column (Length; $30 \mathrm{~m} \times 0.25 \mathrm{~mm}$ ) using a Varian CP 3800 / Tekmar 7000 apparatus (Agilent, CA, USA). In the esophageal tissue, the acetaldehyde and ethanol concentrations were measured by combining four biopsy specimens. Since $0.6 \mathrm{M}$ PCA $300 \mu \mathrm{L}$ was nearly $0.3 \mathrm{~g}$, the ethanol and acetaldehyde concentrations in the esophageal tissue $(C)$ were calculated using the formula:

$$
\begin{gathered}
C=V(\text { value obtained by gas chromatography }) \times \\
\text { (weight of the biopsy specimens }(\mathrm{g})+0.3(\mathrm{~g})) / \\
\text { weight of the biopsy specimens }(\mathrm{g})) .
\end{gathered}
$$

\section{Outcomes}

The primary outcome in this study was the acetaldehyde concentration of the esophageal tissue after drinking alcohol in taking L-cysteine or placebo lozenges. The secondary outcomes were the acetaldehyde concentrations in the saliva, blood and esophageal tissue in taking L-cysteine or placebo lozenges in each $A L D H 2$ genotype. We also evaluated correlations between the acetaldehyde concentration of the esophageal tissue and that of blood or saliva at 60 minutes after drinking. In addition, we investigated the ethanol concentration of each specimen in taking L-cysteine or placebo lozenges in the
$A D H 1 B$ genotypes (active $A D H 1 B * 1 / * 2$ heterozygotes and highlyactive $A D H 1 B * 2 / * 2$ homozygotes).

\section{Genotyping of $A L D H 2$ and $A D H 1 B$}

DNA was extracted from each subject with a DNA Blood Mini Kit (Qiagen, Hilden, Germany). Genotyping for $A L D H 2$ (rs671) and $A D H 1 B$ (rs1229984) was conducted using TaqMan ${ }^{\circledR}$ Drug Metabolism Genotyping Assay (Thermo Fisher Scientific, Waltham, MA, USA).

\section{L-cysteine and placebo lozenges}

Slow-release L-cysteine lozenges contained $3 \mathrm{mg}$ of L-cysteine as the active ingredient. A placebo formulation where L-cysteine was replaced with the same amount of $\mathrm{CaHPO}_{4}$ was prepared following exactly the same method.

\section{Statistical analysis}

Continuous data were presented as means with standard errors of the means (SEM). Student's $t$-test was used to compare the two groups. Comparison between categorical variables was performed using the Fisher's exact test. The mean concentrations of acetaldehyde and ethanol in the saliva and blood were plotted on a graph according to the time after the completion of drinking, and the area under the curve (AUC) from the time before drinking (Time 0) and 30 and 60 minutes after drinking was calculated. For evaluating the relationship between the acetaldehyde concentration of the esophageal tissue and saliva or blood, Pearson's correlation coefficient $(r)$ was calculated. Data processing and statistical analyses were conducted using SPSS version 20.0 for Windows (SPSS, Chicago, IL, USA). A $p$ value of less than 0.05 was regarded as statistically significant.

\section{Results}

Twenty subjects $(10 A L D H 2 * 1 / * 1$ homozygotes and 10 ALDH $2 * 1 / * 2$ heterozygotes) completed the whole procedure, and no adverse event occurred during the study. There was no subject with symptoms suspicious for gastroesophageal reflux disease or reflex esophagitis on EGD. The baseline characteristics of the enrolled subjects are shown in Table 1. There was no significant difference in age and body weight between the $A L D H 2 * 1 / * 1$ homozygotes and $A L D H 2 * 1 / * 2$ heterozygotes. All enrolled subjects continued to take L-cysteine or placebo lozenges during the 60 minutes after drinking. The mean (SEM) numbers of L-cysteine and placebo lozenges taken in the whole procedure were $11.7(1.0)$ and $12.3(1.0)$, respectively.

\section{Acetaldehyde and ethanol concentrations of the esophageal tissue in taking L-cysteine or placebo lozenges}

We evaluated the acetaldehyde and ethanol concentrations of the esophageal tissue in taking L-cysteine or placebo. Typical chromatograms of acetaldehyde and ethanol in the samples and esophageal tissues are shown in Fig. 2A, $\mathrm{B}$, respectively. In these chromatograms, prominent peaks corresponding to acetaldehyde and ethanol were observed. Among cases taking L-cysteine, we could detect acetalde- 
Table 1. Baseline characteristics of the enrolled subjects.

\begin{tabular}{llll}
\hline & $\begin{array}{l}A L D H 2^{*} 1{ }^{*} 1 \\
\text { homozygotes } \\
(n=10)\end{array}$ & $\begin{array}{l}A L D H 2^{*} 1{ }^{*} 2 \\
\text { heterozygotes } \\
(n=10)\end{array}$ & $p$ value \\
\hline $\begin{array}{l}\text { Mean age, years (SEM) } \\
\text { Mean body weight, kg (SEM) }\end{array}$ & $25.6(1.2)$ & $27.9(7.9)$ & 0.39 \\
$\begin{array}{l}\text { Mean number of L-cysteine } \\
\text { lozenges, } n \text { (SEM) }\end{array}$ & $10.5(1.2)$ & $63.3(2.3)$ & 0.77 \\
Mean number of placebo & $11.6(1.2)$ & $12.8(1.4)$ & 0.24 \\
lozenges, $n$ (SEM) & & & \\
$A D H 1 B$ genotype & & 10.0 & 0.48 \\
$A D H 1 B^{*} 2{ }^{*} 2, n$ & 8 & 0 & 0.47 \\
$A D H 1 B^{*} 1{ }^{*} 2, n$ & 2 & 10 & \\
\hline
\end{tabular}

$A L D H$, acetaldehyde dehydrogenase; SEM, standard errors of the means; $A D H$, alcohol dehydrogenase.

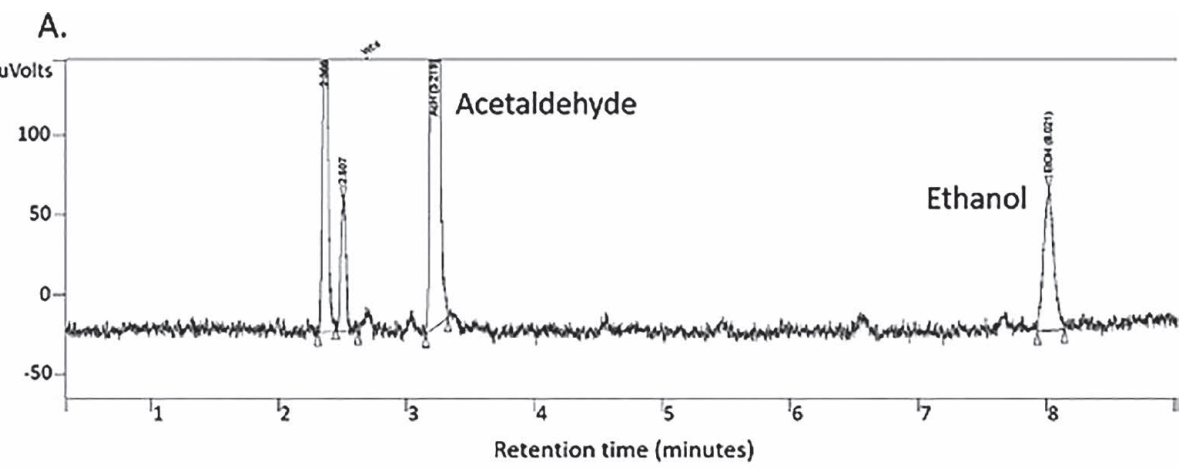

B.

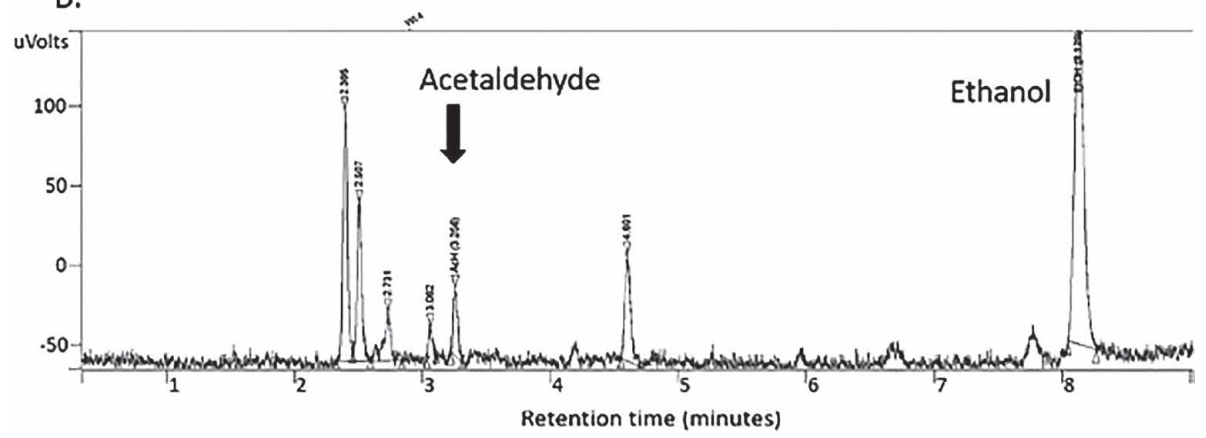

Fig. 2. Typical chromatograms of the esophageal tissue.

A. Chromatogram of the standard acetaldehyde and ethanol.

B. Chromatogram of the esophageal tissue.

Prominent peaks corresponding to acetaldehyde and ethanol were observed in these chromatograms.

hyde from the esophageal tissue in 5 cases, comprising 3 $A L D H 2 * 1 / 1$ homozygotes and $2 A L D H 2 * 1 / * 2$ heterozygotes, whereas acetaldehyde in the esophageal tissue did not appear in any cases taking the placebo (Fig. 3A, B). There was no correlation in the acetaldehyde concentration between the esophageal tissue and saliva or blood at 60 minutes $(r=-0.091, p=0.58$ and $r=-0.12, p=0.46$, respectively).

Concerning the ethanol concentration of the esophageal tissue in each $A D H 1 B$ genotype was shown in Fig. 3C,
D. There was no significant difference between those taking L-cysteine and the placebo in $A D H 1 B * 2 / * 2$ homozygotes. In taking the placebo, the ethanol concentration of the esophageal tissue was significantly higher than that of the saliva and blood at 60 minutes $(p<0.001$ and $p=0.001$, respectively).

Acetaldehyde and ethanol concentrations of the saliva in taking L-cysteine or placebo lozenges

In taking the placebo, the AUC for the acetaldehyde 
A.
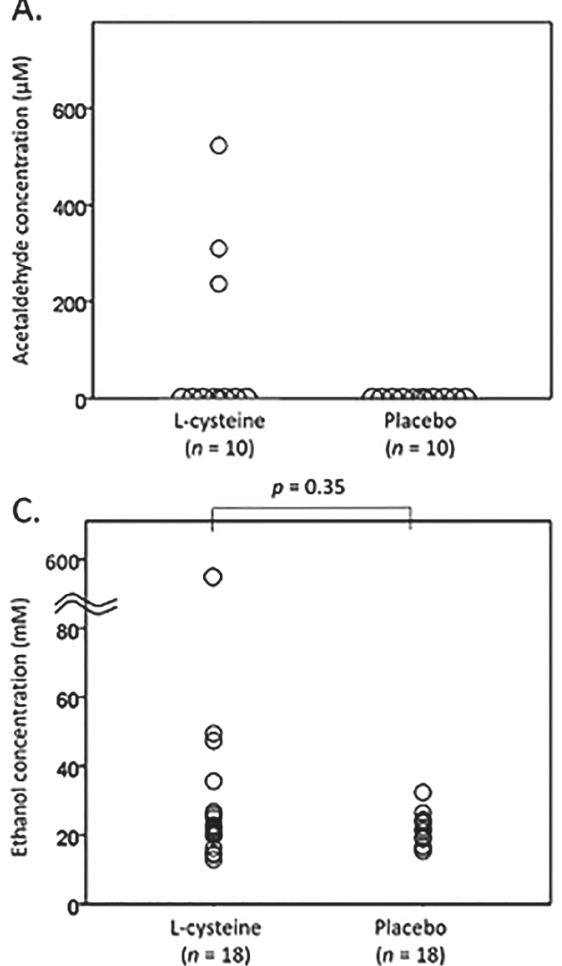

B.

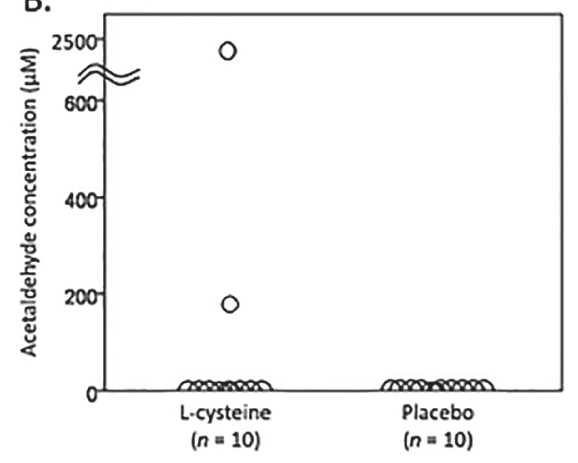

D.

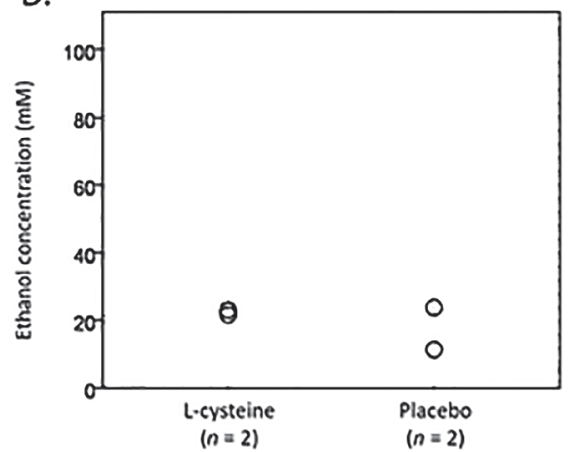

Fig. 3. Acetaldehyde and ethanol concentrations of the esophageal tissue in taking L-cysteine or placebo lozenges.

A. Acetaldehyde concentration in $A L D H 2 * 1 / * 1$ homozygotes $(n=10)$.

B. Acetaldehyde concentration in $A L D H 2 * 1 / * 2$ heterozygotes $(n=10)$.

C. Ethanol concentration in $A D H 1 B * 2 / * 2$ homozygotes $(n=18)$.

D. Ethanol concentration in $A D H 1 B * 1 / * 2$ heterozygotes $(n=2)$.

In taking L-cysteine lozenges, acetaldehyde from the esophageal tissue could be detected in $3 A L D H 2 * 1 / * 1$ homozygotes and $2 A L D H 2 * 1 / * 2$ heterozygotes, whereas acetaldehyde was not detected in any of those taking the placebo. Concerning the ethanol concentration in the esophageal tissue, there was no significant difference between those taking L-cysteine and the placebo lozenges in $A D H 1 B * 2 / * 2$ homozygotes. Statistical analysis was not performed in part due to the small number of samples.

ALDH, acetaldehyde dehydrogenase; $A D H$, alcohol dehydrogenase.

concentration in the saliva tended to be higher in $A L D H 2 * 1 / 2$ heterozygotes than in $A L D H 2 * 1 / * 1$ homozygotes $(p=0.21)$. In addition, a higher AUC for the ethanol concentration in $A D H 1 B * 1 / 2$ heterozygotes was shown ( $p$ $=0.13$ ). In the $A L D H 2 * 1 / * 1$ homozygotes, the acetaldehyde concentration of the saliva in taking L-cysteine was lower than that in taking the placebo at 30 and 60 minutes after drinking (Fig. 4A). The AUC for the acetaldehyde concentration in the saliva was significantly lower in taking L-cysteine than in taking the placebo $(1.7 \pm 0.9(\mu \mathrm{M} \cdot \mathrm{h}$, mean $\pm \mathrm{SEM}$ ) vs. $9.7 \pm 1.5, p=0.002$ ). In addition, similar results in the AUC appeared in the $A L D H 2 * 1 / * 2$ heterozygotes $(7.3 \pm 1.7$ vs. $12.8 \pm 1.8, p=0.036)$ (Fig. 4B). Regarding the ethanol concentration of the saliva (Fig. 4C, $\mathrm{D})$, the AUC in the $A D H 1 B * 2 / * 2$ homozygotes was significantly lower in taking L-cysteine than in taking the placebo $(8.7 \pm 0.8(\mathrm{mM} \cdot \mathrm{h}$, mean $\pm \mathrm{SEM})$ vs. $11.2 \pm 0.8, p=0.022)$.

Acetaldehyde and ethanol concentrations of the blood in taking L-cysteine or placebo lozenges

In taking the placebo, the AUC for the ethanol concen- tration was significantly higher in $A D H 1 B^{*} 1 / *^{2}$ heterozygotes than in $A D H 1 B * 2 / * 2$ homozygotes $(p=0.045$ ), although that for the acetaldehyde concentration in the blood was not different between the two $A L D H 2$ genotypes. The acetaldehyde concentrations of the blood showed no significant differences in the AUC between those taking L-cysteine and the placebo, regardless of the $A L D H 2$ genotypes (Fig. 5A, B). Concerning the ethanol concentration of the blood (Fig. 5C, D), no significant difference in the AUC was shown between those taking the two kinds of lozenges in $A D H 1 B * 2 / 2$ homozygotes.

\section{Discussion}

In this study, we firstly detected acetaldehyde in the esophageal tissue. In addition, although the acetaldehyde concentration of the saliva was significantly lower in those taking L-cysteine lozenges than in those taking the placebo in both $A L D H 2$ genotypes, acetaldehyde of the esophageal tissue appeared only in those taking L-cysteine lozenges.

Acetaldehyde associated with alcohol intake was regarded as a definite carcinogen (a group 1 carcinogen) for 
A.

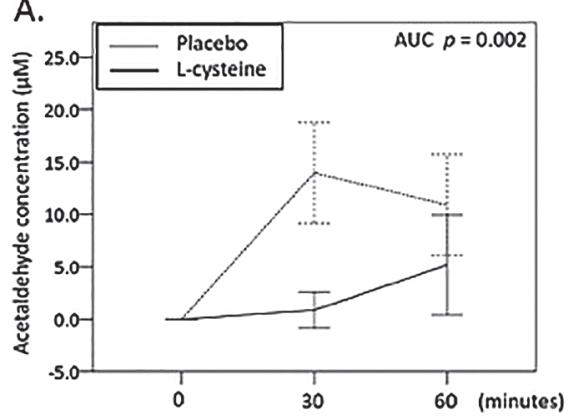

C.

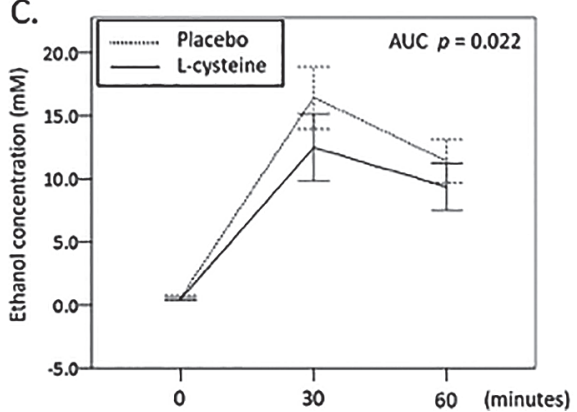

B.

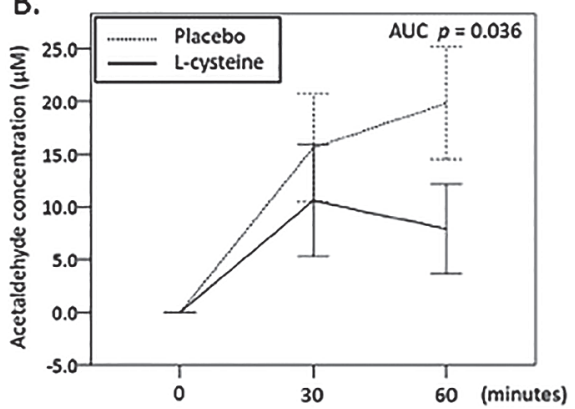

D.

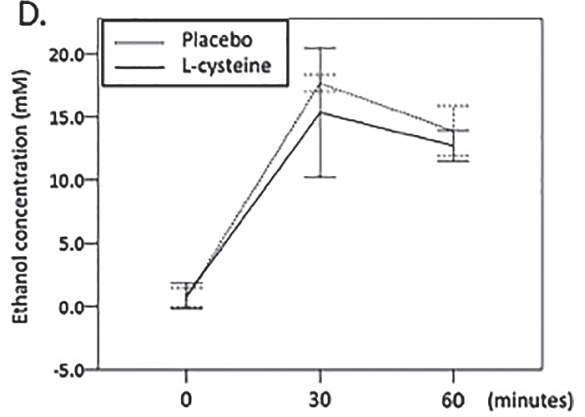

Fig. 4. Acetaldehyde and ethanol concentrations in the saliva in taking L-cysteine or placebo lozenges.

A. Acetaldehyde concentration in $A L D H 2 * 1 / * 1$ homozygotes $(n=10)$.

B. Acetaldehyde concentration in $A L D H 2 * 1 / * 2$ heterozygotes $(n=10)$.

C. Ethanol concentration in $A D H 1 B * 2 / * 2$ homozygotes $(n=18)$.

D. Ethanol concentration in $A D H 1 B * 1 / * 2$ heterozygotes $(n=2)$.

AUC for acetaldehyde concentration in the saliva was significantly lower in taking L-cysteine lozenges than in taking the placebo in both $A L D H 2$ genotypes. Regarding the ethanol concentration of the saliva, the AUC in $A D H 1 B * 2 / * 2$ homozygotes was significantly lower in taking L-cysteine than in taking the placebo. Statistical analysis was not performed in part due to the small number of samples.

$A L D H$, acetaldehyde dehydrogenase; $A D H$, alcohol dehydrogenase; $\mathrm{AUC}$, area under the curve.

the esophagus by the International Agency for Research on Cancer (Secretan et al. 2009). However, its direct measurement in the tissue has remained difficult because of its low levels, high volatility, and rapid metabolism by ALDH (Eriksson et al. 1979). Since acetaldehyde has been shown to interact with DNA to form DNA adducts, such as $N^{2}$-ethylidene-2'-deoxyguanosine (Yukawa et al. 2012), $N^{2}$-ethyl-2'-deoxyguanosine (Matsuda et al. 2006) and 1, $N^{2}$-propano-2'-deoxyguanosine (Yu et al. 2010), a previous study dealing with acetaldehyde in the esophageal tissue used a DNA adduct ( $N^{2}$-ethyl-2'-deoxyguanosine) as a marker for acetaldehyde exposure (Amanuma et al. 2015). Meanwhile, the acetaldehyde concentrations in the human saliva, blood, breath, and gastric juice have been successfully measured (Vakevainen et al. 2001; Muto et al. 2002; Salaspuro et al. 2002; Visapaa et al. 2004; Yokoyama et al. 2008, 2010b; Maejima et al. 2015). In our previous study, for accurately measuring the acetaldehyde concentration, the obtained saliva and gastric juice were transferred into a headspace vial containing PCA to stop microbial acetaldehyde production, and the samples were immediately deep frozen and stored at $-80^{\circ} \mathrm{C}$ to prevent volatilization (Maejima et al. 2015). In this study, we used this technique until transfer on dry ice to the headspace gas chromatogra- phy for measuring the acetaldehyde concentration of the esophageal tissue. In addition, for avoiding the possibility of measuring the acetaldehyde of mucus on the esophageal tissue, mucus on the esophageal mucosa was removed by a sufficient amount of water before taking each biopsy. As a result, we could detect acetaldehyde of the esophageal tissue in 5 cases. To our knowledge, this is the first study measuring the acetaldehyde concentration in the human esophageal tissue, which is one of the clinical implications in this study. This direct measurement of acetaldehyde in the esophageal tissue would contribute to exploring how the acetaldehyde level in the esophageal tissue correlates with DNA damage and carcinogenesis in future studies.

Meanwhile, we found many cases in which acetaldehyde was not detected in the esophageal tissue. In particular, acetaldehyde of the esophageal tissue was not detected in all cases taking the placebo. Several factors such as technical problems and volatilization may have affected the acetaldehyde concentration in the esophageal tissue. Although we believe this study result is reliable because it was performed in a blind manner, a further validation study is needed to confirm our results. In addition, it is desirable to measure parallel samples and calculate the mean value to minimize variations in the samples, although it was ethi- 
A.

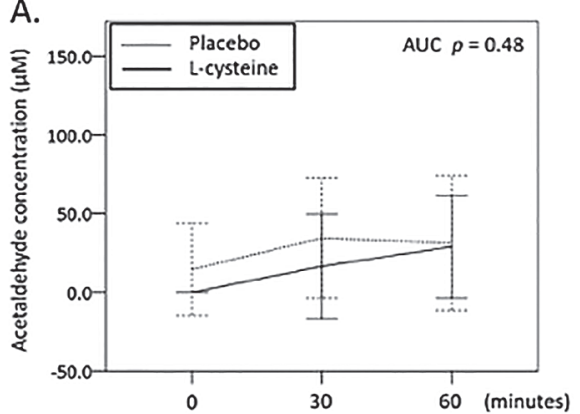

C.

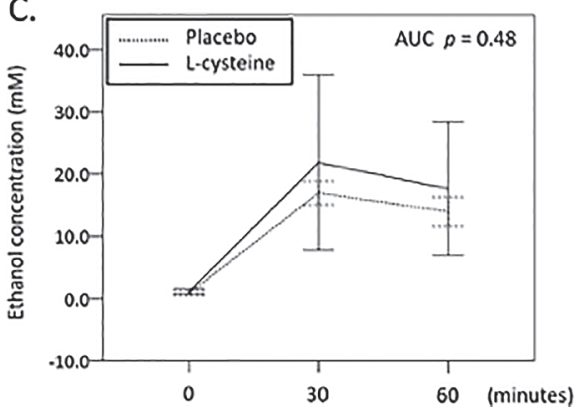

B.
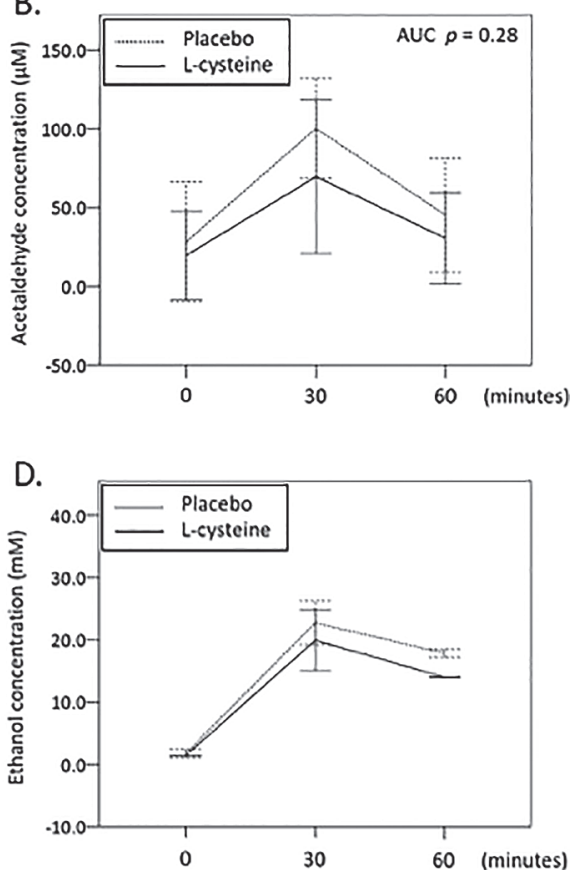

Fig. 5. Acetaldehyde and ethanol concentrations in the blood in taking L-cysteine or placebo lozenges.

A. Acetaldehyde concentration in $A L D H 2 * 1 / * 1$ homozygotes $(n=10)$.

B. Acetaldehyde concentration in $A L D H 2 * 1 / * 2$ heterozygotes $(n=10)$.

C. Ethanol concentration in $A D H 1 B * 2 / * 2$ homozygotes $(n=18)$.

D. Ethanol concentration in $A D H 1 B * 1 / * 2$ heterozygotes $(n=2)$.

There was no significant difference in the AUC for the acetaldehyde concentration between those taking L-cysteine and the placebo lozenges, regardless of the $A L D H 2$ genotype. Similarly, no significant difference in the AUC for the ethanol concentration of blood was shown between the two kinds of lozenges in $A D H 1 B * 2 / 2$ homozygotes. Statistical analysis was not performed in part due to the small number of samples.

$A L D H$, acetaldehyde dehydrogenase; $A D H$, alcohol dehydrogenase; AUC, area under the curve.

cally difficult in this study. Furthermore, one of the reasons for the many cases of undetected acetaldehyde may be that the small concentration of acetaldehyde was below the measurable concentration in the headspace gas chromatography. Recently, Heit et al. (2016) demonstrated the feasibility of headspace gas chromatography mass spectrometry (GC-MS) by measuring the ethanol and acetaldehyde concentrations in the low $\mu \mathrm{M}$ range in murine neuronal tissue. Thus, the headspace GC-MS methods may enable the measurement of acetaldehyde of the esophageal tissue with higher sensitivity.

Regarding the source of acetaldehyde, the esophageal epithelium is exposed to carcinogenic acetaldehyde via an intrinsic and extrinsic pathway (Ohashi et al. 2015). Among these pathways, direct exposure of acetaldehyde, derived from saliva, alcohol beverages, and so on, to the esophageal epithelium has been considered to be the main source of acetaldehyde in the esophageal tissue (Simanowski et al. 1993; Amanuma et al. 2015). In this study, we used slow-release L-cysteine lozenges to reduce acetaldehyde from the saliva. Thus, at the beginning of this study, we hypothesized that the acetaldehyde concentration of the esophageal tissue in taking L-cysteine lozenges would be lower than that in taking placebo lozenges. However, this study revealed that acetaldehyde in the esophageal tissue appeared only in taking L-cysteine lozenges; in contrast, the acetaldehyde concentration of the saliva was significantly lower in taking L-cysteine lozenges than in taking the placebo. In addition, similar results were shown in both ALDH2 genotypes. Although we detected acetaldehyde of the esophageal tissue in a small number of cases, this result is significant because this study was performed in a blind manner. Therefore, there is a possibility that acetaldehyde of the saliva may play a limited role in the acetaldehyde concentration of the esophageal tissue.

In the present study, we also found ethanol in the esophageal tissue in all cases and there was no significant difference in the ethanol concentration between those taking L-cysteine and the placebo in $A D H 1 B * 2 / * 2$ homozygotes. In the esophagus, ADH activity was reported to be approximately 4-fold that of stomach enzymes (Yin et al. 1993). On the other hand, it is controversial whether ALDH2 protein is produced in the esophagus (Yin et al. 1993; Morita et al. 2005; Amanuma et al. 2015). Among these reports, Yin et al. (1993) reported that the ALDH activity in the esophagus was $20 \%$ of that in the stomach. However, Amanuma et al. (2015) demonstrated that ALDH2 production is induced by acetaldehyde in esophageal keratinocytes and 
that ALDH2 plays a protective role against acetaldehydederived DNA damage in esophageal squamous epithelium. Thus, these results in the previous studies and our data suggest that there may be a pathway by which ethanol is metabolized into acetaldehyde, which is further metabolized into acetic acid, in the esophagus, which may affect the acetaldehyde concentration of the esophageal tissue. However, for confirming this hypothesis, further study is needed.

We can raise two strengths in this study. First, this is a prospective, single-blind, placebo-controlled trial using L-cysteine and placebo lozenges, which reduced the bias. In addition, the biopsy specimens of the esophageal tissue were taken by an expert endoscopist who was blinded to any clinical information of the subjects in the same manner, resulting in minimal variance in the sizes of the biopsy specimens. In fact, the weight of the biopsy specimens was similar among the subjects. Therefore, the results related to the acetaldehyde and ethanol concentrations of the esophageal tissue in this study are likely reliable.

This study includes several limitations. First, the dose of L-cysteine and the placebo lozenges depended on the subjects. In this study, the subjects were advised that the lozenges should be kept in their mouths, possibly leading to differences in the doses of L-cysteine and the placebo lozenges among the subjects. This difference may have affected the results of this study. However, there was no correlation between the dose of the L-cysteine lozenges and the acetaldehyde concentration in the saliva, blood, or esophageal tissue (data not shown). Second, we did not measure the baseline acetaldehyde and ethanol concentrations in the esophageal tissue because it is ethically difficult to perform EGD before drinking in each examination. In addition, we did not measure the acetaldehyde and ethanol concentrations of the blood and saliva at the time immediately after drinking. Third, variation of microbes and fungus in each subject may have affected the acetaldehyde concentration because ethanol is oxidized to acetaldehyde by microbial ADH enzyme (Linderborg et al. 2011), although we confirmed that there was no finding suspicious for candida esophagitis on EGD. Finally, we cannot avoid the artefactual formation of acetaldehyde from ethanol in measuring the acetaldehyde concentration of the blood. In fact, the acetaldehyde concentration of the blood in the present study was higher than that in a previous study (Yokoyama et al. 2008), which may have been affected from the artefactual formation of acetaldehyde.

In conclusion, we firstly detected acetaldehyde in human esophageal tissue. In addition, continuous taking of L-cysteine lozenges after drinking may have little effect in reducing acetaldehyde from the esophageal tissue. Instead, intake of L-cysteine lozenges appears to contribute to detection of acetaldehyde in the esophageal tissue.

\section{Acknowledgments}

This study was supported in part by JSPS KAKENHI (15K19308, 15K08945, 17K15917).

\section{Conflict of Interest}

The authors declare no conflict of interest.

\section{References}

Amanuma, Y., Ohashi, S., Itatani, Y., Tsurumaki, M., Matsuda, S., Kikuchi, O., Nakai, Y., Miyamoto, S., Oyama, T., Kawamoto, T., Whelan, K.A., Nakagawa, H., Chiba, T., Matsuda, T. \& Muto, M. (2015) Protective role of ALDH2 against acetaldehyde-derived DNA damage in oesophageal squamous epithelium. Sci. Rep., 5, 14142.

Blonigen, D.M., Timko, C., Moos, B.S. \& Moos, R.H. (2011) Impulsivity is an independent predictor of 15 -year mortality risk among individuals seeking help for alcohol-related problems. Alcohol. Clin. Exp. Res., 35, 2082-2092.

Brooks, P.J., Enoch, M.A., Goldman, D., Li, T.K. \& Yokoyama, A. (2009) The alcohol flushing response: an unrecognized risk factor for esophageal cancer from alcohol consumption. PLoS Med., 6, e50.

Cui, R., Kamatani, Y., Takahashi, A., Usami, M., Hosono, N., Kawaguchi, T., Tsunoda, T., Kamatani, N., Kubo, M., Nakamura, Y. \& Matsuda, K. (2009) Functional variants in ADH1B and ALDH2 coupled with alcohol and smoking synergistically enhance esophageal cancer risk. Gastroenterology, 137, 17681775 .

Eriksson, C.J., Hillbom, M.E. \& Sovijarvi, A. (1979) Difficulties in measuring human acetaldehyde levels. Drug Alcohol Depend., 4, 148.

Heit, C., Eriksson, P., Thompson, D.C., Charkoftaki, G., Fritz, K.S. \& Vasiliou, V. (2016) Quantification of neural ethanol and acetaldehyde using headspace GC-MS. Alcohol. Clin. Exp. Res., 40, 1825-1831.

Homann, N., Jousimies-Somer, H., Jokelainen, K., Heine, R. \& Salaspuro, M. (1997) High acetaldehyde levels in saliva after ethanol consumption: methodological aspects and pathogenetic implications. Carcinogenesis, 18, 1739-1743.

Linderborg, K., Salaspuro, M. \& Vakevainen, S. (2011) A single sip of a strong alcoholic beverage causes exposure to carcinogenic concentrations of acetaldehyde in the oral cavity. Food Chem. Toxicol., 49, 2103-2106.

Maejima, R., Iijima, K., Kaihovaara, P., Hatta, W., Koike, T., Imatani, A., Shimosegawa, T. \& Salaspuro, M. (2015) Effects of ALDH2 genotype, PPI treatment and L-cysteine on carcinogenic acetaldehyde in gastric juice and saliva after intragastric alcohol administration. PLoS One, 10, e0120397.

Matsuda, T., Yabushita, H., Kanaly, R.A., Shibutani, S. \& Yokoyama, A. (2006) Increased DNA damage in ALDH2deficient alcoholics. Chem. Res. Toxicol., 19, 1374-1378.

Morita, M., Oyama, T., Kagawa, N., Nakata, S., Ono, K., Sugaya, M., Uramoto, H., Yoshimatsu, T., Hanagiri, T., Sugio, K., Kakeji, Y. \& Yasumoto, K. (2005) Expression of aldehyde dehydrogenase 2 in the normal esophageal epithelium and alcohol consumption in patients with esophageal cancer. Front. Biosci., 10, 2319-2324.

Muto, M., Nakane, M., Hitomi, Y., Yoshida, S., Sasaki, S., Ohtsu, A., Yoshida, S., Ebihara, S. \& Esumi, H. (2002) Association between aldehyde dehydrogenase gene polymorphisms and the phenomenon of field cancerization in patients with head and neck cancer. Carcinogenesis, 23, 1759-1765.

Ohashi, S., Miyamoto, S., Kikuchi, O., Goto, T., Amanuma, Y. \& Muto, M. (2015) Recent advances from basic and clinical studies of esophageal squamous cell carcinoma. Gastroenterology, 149, 1700-1715. 
Salaspuro, M. (2011) Acetaldehyde and gastric cancer. J. Dig. Dis., 12, 51-59.

Salaspuro, V., Hietala, J., Kaihovaara, P., Pihlajarinne, L., Marvola, M. \& Salaspuro, M. (2002) Removal of acetaldehyde from saliva by a slow-release buccal tablet of L-cysteine. Int. J. Cancer, 97, 361-364.

Salaspuro, V.J., Hietala, J.M., Marvola, M.L. \& Salaspuro, M.P. (2006) Eliminating carcinogenic acetaldehyde by cysteine from saliva during smoking. Cancer Epidemiol. Biomarkers Prev., 15, 146-149.

Secretan, B., Straif, K., Baan, R., Grosse, Y., El, Ghissassi, F., Bouvard, V., Benbrahim-Tallaa, L., Guha, N., Freeman, C., Galichet, L. \& Cogliano, V.; WHO International Agency for Research on Cancer Monograph Working Group (2009) A review of human carcinogens-Part E: tobacco, areca nut, alcohol, coal smoke, and salted fish. Lancet Oncol., 10, 10331034.

Simanowski, U.A., Suter, P., Stickel, F., Maier, H., Waldherr, R., Smith, D., Russell, R.M. \& Seitz, H.K. (1993) Esophageal epithelial hyperproliferation following long-term alcohol consumption in rats: effects of age and salivary gland function. J. Natl. Cancer Inst., 85, 2030-2033.

Sprince, H., Parker, C.M., Smith, G.G. \& Gonzales, L.J. (1975) Protective action of ascorbic acid and sulfur compounds against acetaldehyde toxicity: implications in alcoholism and smoking. Agents Actions, 5, 164-173.

Tanaka, F., Yamamoto, K., Suzuki, S., Inoue, H., Tsurumaru, M., Kajiyama, Y., Kato, H., Igaki, H., Furuta, K., Fujita, H., Tanaka, T., Tanaka, Y., Kawashima, Y., Natsugoe, S., Setoyama, T., et al. (2010) Strong interaction between the effects of alcohol consumption and smoking on oesophageal squamous cell carcinoma among individuals with ADH1B and/or ALDH2 risk alleles. Gut, 59, 1457-1464.

Vakevainen, S., Tillonen, J. \& Salaspuro, M. (2001) 4-Methylpyrazole decreases salivary acetaldehyde levels in aldh2-deficient subjects but not in subjects with normal aldh2. Alcohol. Clin. Exp. Res., 25, 829-834.

Visapaa, J.P., Gotte, K., Benesova, M., Li, J., Homann, N., Conradt, C., Inoue, H., Tisch, M., Horrmann, K., Vakevainen, S., Salaspuro, M. \& Seitz, H.K. (2004) Increased cancer risk in heavy drinkers with the alcohol dehydrogenase $1 \mathrm{C}^{*} 1$ allele, possibly due to salivary acetaldehyde. Gut, 53, 871-876.

Yin, S.J., Chou, F.J., Chao, S.F., Tsai, S.F., Liao, C.S., Wang, S.L., Wu, C.W. \& Lee, S.C. (1993) Alcohol and aldehyde dehydrogenases in human esophagus: comparison with the stomach enzyme activities. Alcohol. Clin. Exp. Res., 17, 376-381.

Yokoyama, A., Kamada, Y., Imazeki, H., Hayashi, E., Murata, S., Kinoshita, K., Yokoyama, T. \& Kitagawa, Y. (2016) Effects of ADH1B and ALDH2 genetic polymorphisms on alcohol elimination rates and salivary acetaldehyde levels in intoxicated Japanese alcoholic men. Alcohol. Clin. Exp. Res., 40, 12411250.

Yokoyama, A. \& Omori, T. (2005) Genetic polymorphisms of alcohol and aldehyde dehydrogenases and risk for esophageal and head and neck cancers. Alcohol, 35, 175-185.

Yokoyama, A., Omori, T. \& Yokoyama, T. (2010a) Alcohol and aldehyde dehydrogenase polymorphisms and a new strategy for prevention and screening for cancer in the upper aerodigestive tract in East Asians. Keio J. Med., 59, 115-130.

Yokoyama, A., Tsutsumi, E., Imazeki, H., Suwa, Y., Nakamura, C., Mizukami, T. \& Yokoyama, T. (2008) Salivary acetaldehyde concentration according to alcoholic beverage consumed and aldehyde dehydrogenase-2 genotype. Alcohol. Clin. Exp. Res., 32, 1607-1614.

Yokoyama, A., Tsutsumi, E., Imazeki, H., Suwa, Y., Nakamura, C. \& Yokoyama, T. (2010b) Polymorphisms of alcohol dehydrogenase-1B and aldehyde dehydrogenase-2 and the blood and salivary ethanol and acetaldehyde concentrations of Japanese alcoholic men. Alcohol. Clin. Exp. Res., 34, 1246-1256.

Yu, H.S., Oyama, T., Isse, T., Kitagawa, K., Pham, T.T., Tanaka, M. \& Kawamoto, T. (2010) Formation of acetaldehyde-derived DNA adducts due to alcohol exposure. Chem. Biol. Interact., 188, 367-375.

Yukawa, Y., Muto, M., Hori, K., Nagayoshi, H., Yokoyama, A., Chiba, T. \& Matsuda, T. (2012) Combination of ADH1B*2/ ALDH2 $* 2$ polymorphisms alters acetaldehyde-derived DNA damage in the blood of Japanese alcoholics. Cancer Sci., 103, 1651-1655.

Zakhari, S. (2006) Overview: how is alcohol metabolized by the body? Alcohol Res. Health, 29, 245-254. 